\title{
Computerassistierte Osteosynthesen am Becken
}

\author{
Ulrich Stöckle, Benjamin König, Norbert Haas
}

\section{Zusammenfassung}

Navigationsverfahren auf der Basis von CT-Daten werden seit 1994 in der Wirbelsäulenchirurgie eingesetzt. In vielen Studien konnte eine Erhöhung der Präzision nachgewiesen werden, so dass heutzutage in vielen Kliniken die navigationsgestützte Pedikelschraubenplatzierung ein Routineverfahren darstellt. Ausgehend von der Erfahrung in der Wirbelsäulenchirurgie wurde zunächst die CT-gestützte Navigation bei wenig dislozierten Beckenring- und Azetabulumfrakturen eingesetzt für perkutane Schraubenosteosynthesen. Nach experimentellen Vorstudien erfolgten dann auch 25 perkutane Schraubenosteosynthesen mit der bildwandlergestützten Navigation. Die postoperative Kontrolle der Schraubenlage er- folgte jeweils mittels postoperativem Röntgen und CT. Insgesamt konnten 29 der 30 eingebrachten Schrauben korrekt platziert werden. Bei einer Schraube fiel im postoperativen CT eine ventrale Kortikalisperforation im Sakrum auf, aber ohne jegliche neurologische Komplikation. Aufgrund dieser ersten klinischen Erfahrungen wird die Indikation für die CT-gestützte Navigation vor allem bei wenig dislozierten Azetabulumfrakturen oder SI-Verschraubungen bei Sakrumdysplasie gesehen, während die bildwandlergestützte Navigation sich bei entsprechender Bildqualität als Verfahren der Wahl für SI-Verschraubungen bei traumatischen oder degenerativen Instabilitäten bewährt hat, vor allem wenn Repositionsmaneuver erforderlich sind.

\section{Einleitung}

In der Becken- und Azetabulumchirurgie wird aufgrund der nicht unerheblichen Zugangsmorbidität eine Reduktion der erweiterten operativen Zugänge angestrebt. Hierbei soll aber weiterhin die stufenfreie Rekonstruktion des Azetabulums und die möglichst anatomische Wiederherstellung des dorsalen Beckenringes das Ziel sein.

Perkutane Schraubenosteosynthesen im Beckenbereich sind jedoch aufgrund der komplexen Beckengeometrie technisch anspruchsvoll. Zur Stabilisierung von Beckenring- oder Azetabulumfrakturen

OP-JOURNAL 2002; 17: 16-20

(c) Georg Thieme Verlag Stuttgart · New York werden sie zumeist unter Bildwandlerkontrolle eingebracht. Am häufigsten wird die perkutane Stabilisierung des SI Gelenkes bei entsprechenden Verletzungen durchgeführt. Das Vorgehen unter fluoroskopischer Kontrolle erfordert hierbei zum Teil erhebliche Durchleuchtungszeiten, da immer nur in einer Projektion gebohrt werden kann und dann in den anderen Projektionen die Lage kontrolliert wird. Nicht selten sind mehrere Bohrungen erforderlich mit wieder- holten Durchleuchtungen in unterschiedlichen Projektionen, so dass Durchleuchtungszeiten von bis zu 10 min pro Schraube resultieren können [11].

Navigationsverfahren auf der Basis von CT-Daten haben sich vor allem in der Wirbelsäulenchirurgie bewährt. Bei reduzierter Strahlenexposition kann die Präzision gesteigert werden [3]. Im eigenen Vorgehen konnten zwischen Mai und Dezember 2000 an der thorakalen Wirbelsäule von 126 Pedikelschrauben 124 korrekt platziert werden [13]. Aufgrund dieser guten Erfahrungen wurde die CT-gestützte Navigation auch in der Beckenchirurgie angewandt. Nach entsprechenden experimentellen Studien mit Definition der erforderlichen Bildwandlerprojektionen [12] wurde auch die neue Methode der bildwandlergestützten Navigation klinisch eingesetzt.

Im Folgenden soll über die ersten klinischen Erfahrungen sowohl mit der CT-gestützten als auch der BV-gestützten Navigation in der Beckenchirurgie berichtet werden. Ein besonderer Schwerpunkt liegt hierbei auf der Indikationsstellung für das jeweilige Verfahren und den Grenzen.

\section{Material und Methode}

Im Zeitraum zwischen 1. Juni 2000 und 31. Dezember 2001 wurden bei 25 Patienten insgesamt 30 perkutane Schraubenosteosynthesen im Beckenbereich mit einem der Navigationsverfahren durchgeführt (Tab.1).
Tab. 1 Verteilung der Indikationen für die navigierten Schraubenpositionen

\begin{tabular}{llllr} 
& $\begin{array}{l}\text { Azetabulum- } \\
\text { fraktur }\end{array}$ & $\begin{array}{l}\text { Beckenring- } \\
\text { verletzung }\end{array}$ & SI-Arthrose & $\mathrm{n}$ \\
\hline CT-gestützte Navigation & 3 & 1 & 1 & 5 \\
BV-gestützte Navigation & 4 & 15 & 6 & 25 \\
$\mathrm{n}$ & 7 & 16 & 7 & 30
\end{tabular}




\section{CT-gestützte Navigation}

Es wurde das Navigationssystem der Firma Medivision ${ }^{\circledR}$ verwendet. Die mit $2 \mathrm{~mm}$ Schichtdicke akquirierten CT-Daten wurden in die Workstation eingelesen. Entsprechend der Frakturlokalisation erfolgten die Definition der Referenzpunkte für das Matching und die Planung der Schraubenposition. Für das Paired Point matching werden fünf Punkte benötigt. Diese wurden anhand der 3D-Rekonstruktion bei der präoperativen Planung festgelegt. Bei perkutanen Verfahren empfiehlt es sich, die Punkte entlang des Beckenkammes zu legen, der in der Regel leicht zugänglich ist, da dann nur kleine zusätzliche Hautinzisionen erforderlich sind. Neben der leicht tastbaren Spina iliaca anterior superior haben sich Punkte in definiertem Abstand dazu auf dem Beckenkamm und knöcherne Vorsprünge bewährt, die gut aufzusuchen sind. Spezielle Markerschrauben wurden nicht verwendet. Für das Oberflächenmatching wurden insgesamt zwischen 15 und 20 Punkte mit dem Pointer eingegeben. Diese Punkte lagen in der Umgebung der zuvor aufgesuchten Punkte auf dem Beckenkamm und im eigentlichen operativen Bereich. Nach erfolgreichem Matching sowie entsprechender Verifikation wurde mit dem navigierten Bohren begonnen.

Während des gesamten operativen Eingriffs war der Bildwandler im OP-Setup, um die Position des Bohrers bzw. Führungsdrahtes zu kontrollieren.

\section{BV-gestützte Navigation}

Es wurde ein herkömmlicher Röntgenbildverstärker der Firma Ziehm ${ }^{\circledR}$ verwendet und das C-Arm-Navigations-Modul der Firma Medivision ${ }^{\circledR}$. Optoelektronische Marker sind hierbei an den Instrumenten, der Patientenreferenz und dem C-Arm montiert, um über eine Infrarotkamera (Fa. Optotrak ${ }^{\circledR}$ ) die Position im Raum zu erfassen. Es ist möglich, bis zu vier Bildwandlerprojektionen simultan auf einem Monitor anzuzeigen. Die Richtung und Länge eines referenzierten Bohrers werden in die zuvor aufgenommenen C-Arm-Bilder eingeblendet. Ohne erneutes Röntgen kann so der Bohrvorgang in allen vier Projektionen gleichzeitig kontrolliert werden.

Die im Rahmen einer Versuchsreihe an Kunststoffbecken (Fa. Synthes ${ }^{\circledR}$ ) definierten Bildwandlerprojektionen für Beckenschrauben [12] wurden auch im klini- schen Einsatz verwendet. Die Bildwandlerdarstellungen stellen hierbei die knöchernen Begrenzungen der Schraubenkanäle dar und wichtige anatomische Strukturen wie das Azetabulum. Unter Berücksichtigung bereits vorhandener Standardprojektionen sind sie gut reproduzierbar.

\section{OP-Technik}

Es wurden jeweils kanülierte 7,3-mmSchrauben mit einem 2,8-mm-Führungs-
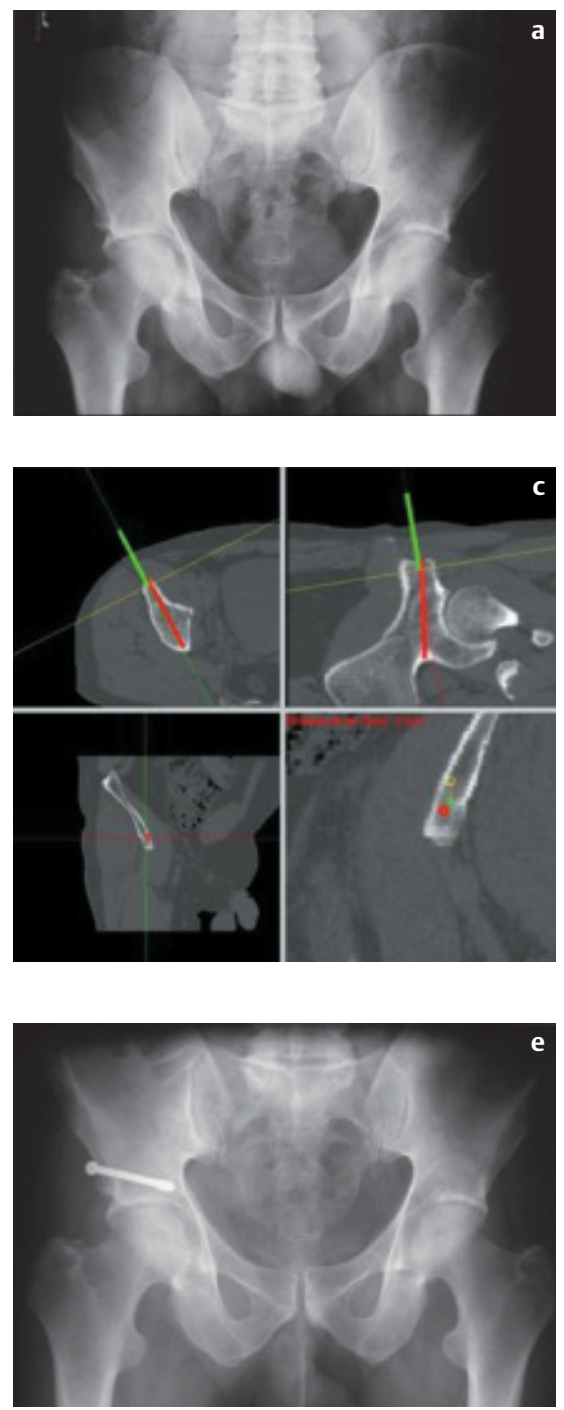

Abb.1a-f CT-navigierte Verschraubung einer vorderen Pfeilerfraktur. Die Stabilisierung erfolgte, um eine frühzeitige Mobilisierung des Patienten zu ermöglichen. Am dritten postoperativen Tag konnte der Patient aus der stationären Behandlung entlassen werden. (a, b) konventionelle Röntgenbeckenübersicht und CT. (c, d) OP-Setup mit referenzierter Bohrmaschine, Monitoransicht. (e, f) Postop. Röntgen und CT-Kontrolle im Vergleich zur Planung (rote Linie).

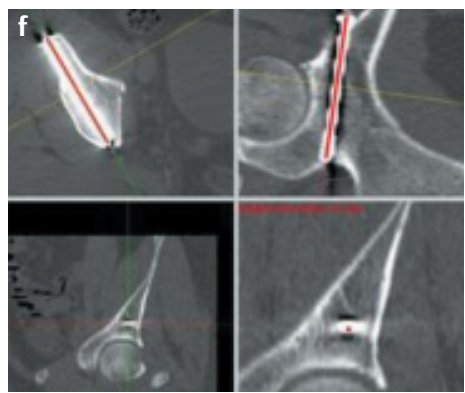

draht verwendet. Nachdem die vorklinischen Versuchsreihen gezeigt hatten, dass selbst der 2,8-mm-Führungsdraht nicht unerhebliche Abweichungen von dem geplanten Verlauf aufweist, wurde die Technik folgendermaßen für beide Verfahren modifiziert:

Nach erfolgreichem Matching bzw. Speichern der geeigneten Bildwandlerprojektionen wurde zunächst mit dem referenzierten Pedikelfinder aus dem Wirbelsäuleninstrumentarium der richtige Haut-
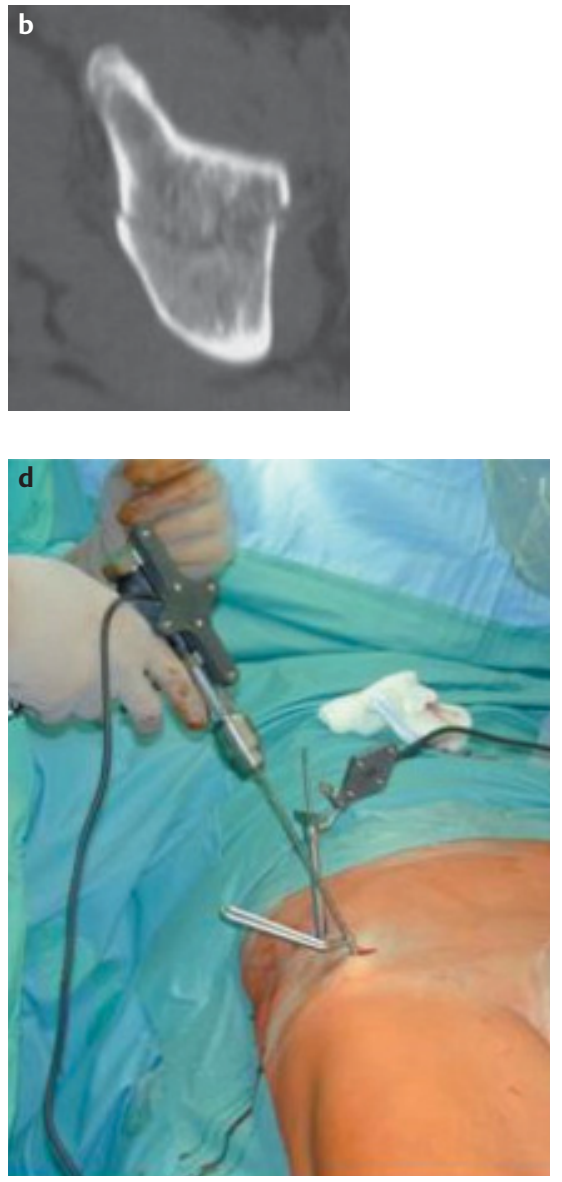
eintrittspunkt lokalisiert. Nach Hautinzision und stumpfer Präparation auf die Knochenoberfläche wurde dann auch der Knocheneintrittspunkt mit dem Pedikelfinder markiert. Mit einem speziell verstärkten kanülierten Bohrer wurde dann mit der referenzierten Bohrmaschine das Bohrloch angelegt, im Falle der SISchrauben bis knapp über die SI-Fuge hinaus. Daraufhin wurde der Führungsdraht durch den kanülierten Bohrer geschoben und nach erneuter Lagekontrolle im BV in den erforderlichen Ebenen die Schraube eingebracht.

Um die Präzision der Verfahren zu erfassen, wurde die Lage der jeweils eingebrachten Schrauben im CT kontrolliert. Bei bildwandlergestützter Navigation wurden intraoperative BV-Bilder, Monitoransichten und postoperative Röntgenbilder verglichen.

\section{Ergebnisse}

\section{CT-gestuitzte Navigation (Abb.1)}

Mit CT-gestützter Navigation wurden zweimal Schraubenosteosynthesen bei kaum dislozierten Azetabulumfrakturen durchgeführt. Hierbei handelte es sich um eine vordere Pfeiler- und eine Querfraktur. Bei der vorderen Pfeilerfraktur wurde eine Schraube eingebracht, bei der Querfraktur zwei. Das Matching war jeweils auf Anhieb erfolgreich, wobei zwei zusätzliche kleine Inzisionen am Beckenkamm notwendig waren. Es zeigte sich jedoch bei der Verifikation, dass nur dann navigiert werden konnte, wenn alle Punkte des Surface Matching auf der zu navigierenden Seite lagen. Waren die Punkte auch auf den gegenseitigen Beckenkamm verteilt, stimmte die Navigationsanzeige trotz guter Matchingwerte nicht exakt mit der intraoperativen Bildwandlerkontrolle überein.

Die Lage der navigierten Schrauben war jeweils korrekt und entsprach exakt der präoperativen Planung (Abb.1c).

Zweimal erfolgten perkutane SI-Verschraubungen mit CT-gestützter Navigation. Die Indikationen waren eine sakro-iliakale Luxationsfraktur und eine SI-Arthrose.

Auch bei den SI-Schrauben war das Matching jeweils auf Anhieb möglich, bei ebenfalls zwei zusätzlichen Inzisionen am Beckenkamm. Die Positionierung der Schrauben war identisch mit der Planung.

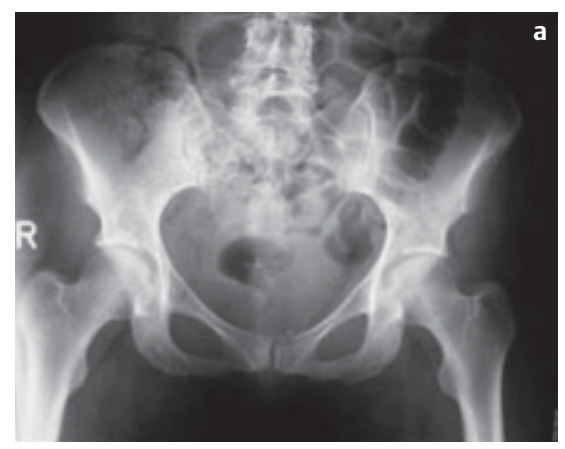

b
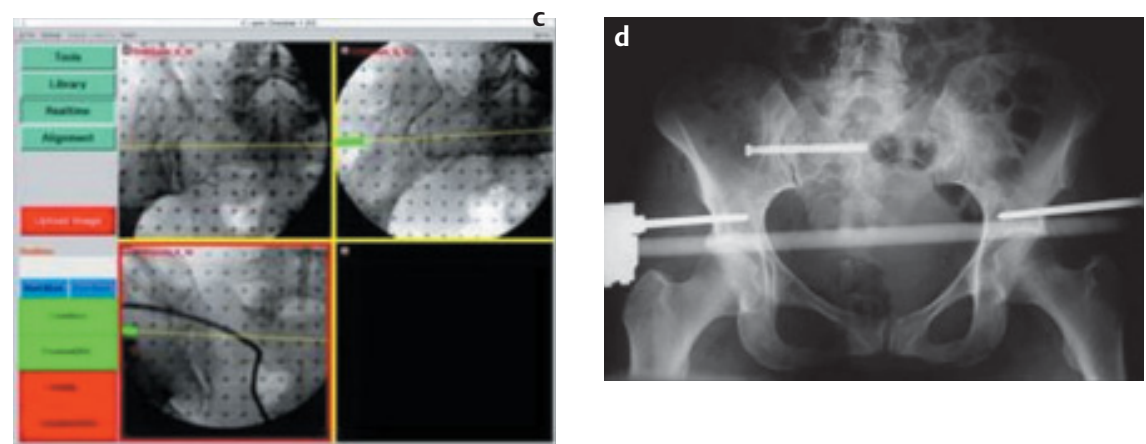

Abb.2a-d Instabile Beckenverletzung mit Sakrumfraktur re (C1.3). (a) Röntgen-Beckenübersicht. (b) CT. (c) Intraoperative Monitoransicht zur SI-Schraubenosteosynthese re. als Stellschraube nach Distraktion über den Fixateur. (d) Postoperatives Röntgenbild.

\section{Bildwandlergestützte Navigation (Abb. 2 u. 3)}

Mit der BV-gestützten Navigation wurden 4 perkutane Schraubenosteosynthesen bei Azetabulumfrakturen eingebracht und 21 SI-Schrauben, davon 20 in S1 und eine in S2. In einem Fall fiel erst postoperativ die ungenügende Reposition im hinteren Beckenring auf bei sakroiliacaler Luxationsfraktur. Durch die verminderte Bildqualität mit den Navigationsmarkern war dies intraoperativ nicht entsprechend erkannt worden. Die erforderliche Revision ist dann auf konventionelle Weise erfolgt. Bei einer Patientin mit transforaminaler Sakrumfraktur beidseits, bei der beidseits die Stabilisierung mit perkutanen SI-Schrauben erfolgte, lagen die Schrauben zwar in der konventionellen Röntgen-Kontrolle (Inlet- und OutletProjektion) korrekt, in der CT-Kontrolle zeigte sich jedoch auf einer Seite eine ventrale Perforation der Sakrumkortikalis. Bei ansonsten korrekter Reposition ohne jegliche neurologische Komplikation wurde die Schraube belassen (Abb. 3).

Bis auf diese eine Fehllage waren 24 der 25 mit BV-gestützter Navigation eingebrachten Schrauben sowohl in der Röntgen- als auch CT-Kontrolle korrekt plat- ziert. Die durchschnittliche Durchleuchtungszeit betrug 0,5 min pro Schraube.

\section{Diskussion}

Die häufigste Anwendung von perkutanen Schraubenosteosynthesen im Beckenbereich stellen Verschraubungen des SI-Gelenkes dar bei wenig dislozierten Sakrumfrakturen oder SI-Gelenksprengungen, die geschlossen reponiert werden können $[1,8-10]$. Aber auch bei Azetabulumfrakturen werden bei geeigneter Frakturform und möglicher geschlossener Reposition zunehmend perkutane Schraubenosteosynthesen durchgeführt $[2,7,11]$. Bei gering dislozierten Azetabulumfrakturen ist hierbei das Ziel die frühzeitige Mobilisierung des Patienten. Aufgrund der komplexen Beckengeometrie sind perkutane Schraubenosteosynthesen technisch anspruchsvoll. Bisher werden vornehmlich kanülierte Schrauben unter Bildwandlerkontrolle eingebracht. Da die erforderlichen Bildwandler-Projektionen nur separat kontrolliert werden können, ist nicht selten ein mehrfaches Korrigieren des Führungsdrahtes erforderlich bis zur gewünschten Positionierung. In der Literatur wird für diese wiederholten Projektionswechsel eine durchschnittliche Durchleuchtungszeit zwischen 1,4 und 

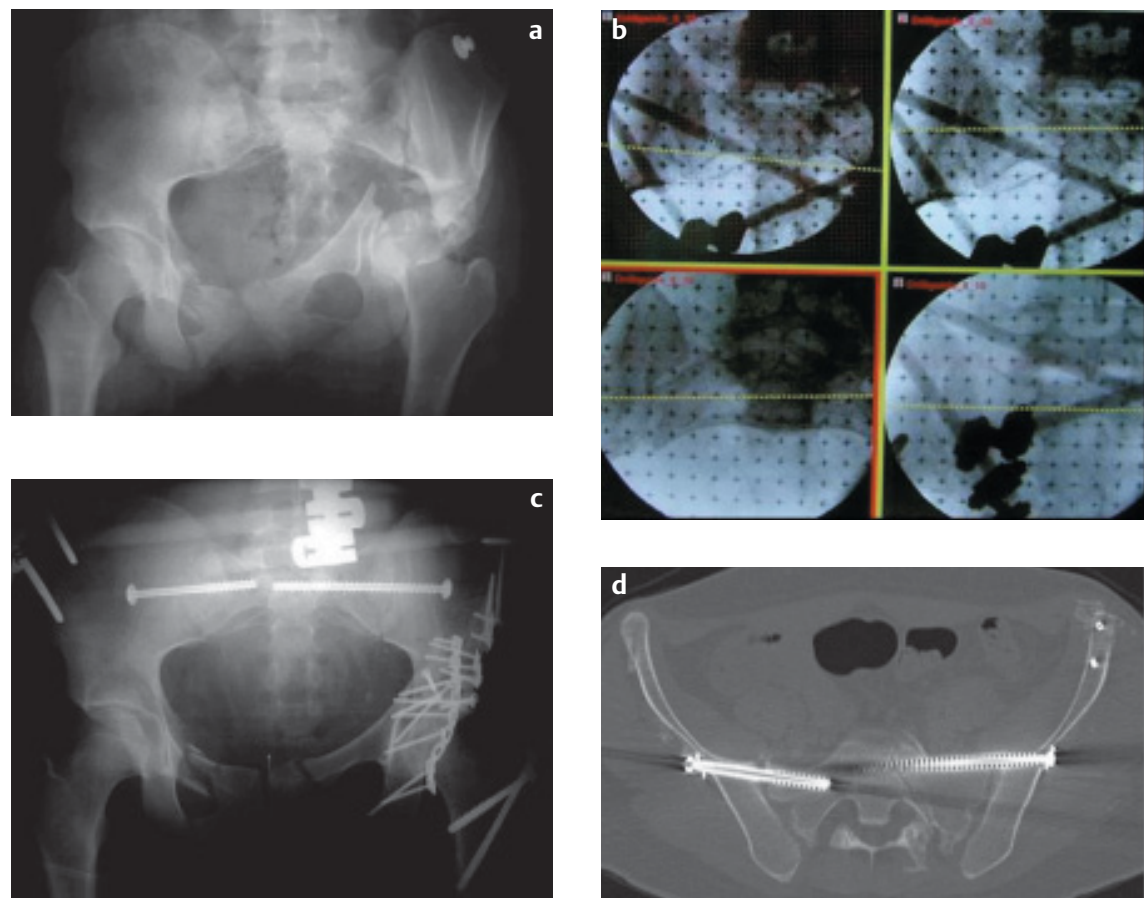

Abb. 3a-d Polytraumatisierte Patientin mit instabiler Beckenringverletzung mit Sakrumfraktur beidseits und Azetabulum-2-Pfeilerfraktur. (a) Röntgen-Beckenübersicht. (b) BV-Monitoransichten. (c) Postoperatives Röntgen und CT. Ventrale Kortikalisperforation links ohne neurologische Komplikation.

4,4 Minuten pro SI-Schraube angegeben $[8,9]$. In Einzelfällen wird sogar über bis zu 10 Minuten Durchleuchtungszeit pro Schraube berichtet [11].

CT-kontrollierte Verschraubungen erlauben bei entsprechender Qualität des CT mittels CT-gestützter Navigation das Einbringen der Schrauben mit hoher Präzision $[1,2,6]$.

Bei nicht oder nur gering dislozierten Frakturen bzw. bei Frakturen, die geschlossen reponiert und retiniert werden können, ist so eine exakte Schraubenplatzierung möglich. Das Matchingverfahren kann sich jedoch aufwändig gestalten, da das knöcherne Becken von reichlich Weichteilen umgeben ist. Entweder können Markerschrauben verwendet werden, die bereits vor dem Navigations-CT eingebracht werden, oder es müssen anhand des CT markante Punkte entlang des Beckenkamms für das Matching ausgewählt werden, die über kleine Stichinzisionen wiedergefunden werden können. Sind Repositionsmaneuver erforderlich, muss nach jeder Reposition ein neuer Datensatz akquiriert werden, so dass entweder ein CT im OP vorhanden sein sollte oder die OP im CT stattfindet. Dies ist nicht selten mit logistischen Problemen verbunden.
Die Bildwandler-gestützte Navigation ist ein neues Verfahren, bei dem Bildwandler, Patient und die verwendeten Instrumente mit optoelektronischen Markern referenziert werden und über Infrarotkameras die jeweilige Position im Raum erfasst wird [4,5]. Es hat den Vorteil, dass ein Bildwandler bereits im unfallchirurgischen OP vorhanden ist und häufig genutzt wird. Bei der bildwandlergestützten Navigation werden die erforderlichen Bildwandlerprojektionen, die sonst mehrfach separat eingestellt werden, nur einmal zu Beginn der Intervention aufgenommen, um dann gleichzeitig auf dem Navigationsmonitor dargestellt zu werden.

Eine gute Qualität der Bildwandlerdarstellung in den definierten Projektionen ist unabdingbare Voraussetzung für ein sicheres und präzises Einbringen der Schrauben. Reicht die durch den Navigationsaufsatz verminderte Bildqualität nicht aus, die Repositionsqualität suffizient zu beurteilen, sollte das Verfahren auf konventionelle Art ohne den speziellen Navigationsaufsatz erfolgen.

Sind die erforderlichen Bildwandlerprojektionen gut zu beurteilen, ist mit der bildwandlergestützten Navigation eine sichere und präzise Schraubenplatzierung möglich.

Mit durchschnittlich 0,5 min Durchleuchtungszeit pro Schraube konnte in unserer Serie auch die Strahlenexposition für Patient und Operateur gegenüber den bekannten Werten aus der Literatur $[8,9]$ deutlich geringer gehalten werden. Bei Sakrumdysplasie jedoch, die anhand des CT gut erfasst werden kann, sollte die CT-gestützte Navigation vorgezogen werden (Tab.2).

Sowohl bei der CT-gestützten Navigation als auch bei der BV-gestützten Navigation sollte der Bildwandler im OP-Setup verbleiben.

Das Navigationssystem ist eine zusätzliche Hilfe, um die Präzision einzelner Operationsverfahren zu erhöhen und gleichzeitig die Strahlenexposition zu reduzieren. Allein die Verwendung eines Navigationsverfahrens kann jedoch auch bei guten Matchingwerten keine 100\%ige Präzision gewährleisten. Durch unbemerkte Manipulationen an der Referencebase z.B. kann die gesamte Darstellung verschoben werden. Während auf dem Navigationsbildschirm immer die virtuelle Realität dargestellt ist, zeigt der Bildwandler jedoch die reale Situation im OP. Um grobe Fehlplatzierungen zu vermeiden, wie sie von anderen Arbeitsgruppen beschrieben wurden [3], hat es sich deshalb bewährt, den Bildwandler im OP-Setup zu belassen und einzelne $\mathrm{Ar}$ -

Tab.2 Eigenschaften und Indikationen der Navigationsverfahren in der Beckenchirurgie

\begin{tabular}{lll} 
& CT-gestützte Navigation & BV-gestützte Navigation \\
\hline \multirow{2}{*}{ Vorteile } & - präoperative Planung & - BV verfügbar \\
& - Schnittbilder & - kein Matching \\
\multirow{3}{*}{ Nachteile } & - 3D-Darstellung & - jederzeit Update \\
& - kufwändiges Matching & - keine 3D-Schnittebenen \\
Indikation & - Nicht oder nur gering dislozierte & - - Fegrenzte Bildqualität \\
& Frakturen & sition \\
& - Dysplasieformen & - standardisierte Projektionen
\end{tabular}


beitsschritte mit einem Kontrollbild in zumindest einer Ebene zu dokumentieren.

Insgesamt sind die Ergebnisse der bildwandlergestützten Navigation bei der technisch anspruchsvollen Anwendung der perkutanen Beckenverschraubung mit 29 von 30 korrekt eingebrachten Schrauben viel versprechend. Bei einer durchschnittlichen Durchleuchtungszeit von 0,5 Minuten/Schraube konnte eine hohe Präzision der eingebrachten Schrauben erreicht werden. Mit weiteren Verbesserungen der Bildwandlerqualität und den erwähnten technischen Änderungen sollte eine weitere Verbesserung der Präzision möglich sein. Dann wird das Verfahren der bildwandlergestützten Navigation nicht nur für Beckenverschraubungen, sondern für den klinischen Einsatz in der gesamten Unfallchirurgie sehr hilfreich sein.

Inwiefern die Anbindung des neuen 3DIso-C-Bildwandlers der Fa. Siemens an die Navigation durch die Möglichkeit der Schnittbilder und Rekonstruktionen die Vorteile der BV-gestützten Navigation und der CT-gestützten Navigation in sich vereint, müssen zukünftige klinische Studien zeigen.

\section{Literatur}

${ }^{1}$ Ebrahim NA et al. Percutaneous computed tomography-guided stabilization of posterior pelvic fractures. Clin Orthop 1994; 307: $222-8$

${ }^{2}$ Gay SB et al. Percutaneous screw fixation of acetabular fractures with CT-guidance: preliminary results of a new technique. Am J Roentgenol 1992; 158(4): 819-22

${ }^{3}$ Gebhard F, Kinzl L, Arand M. Computerassistierte Chirurgie. Unfallchirurg 2000; 103: $612-17$

${ }^{4}$ Hamadeh A et al. Automated 3-dimensional computed tomographic and fluoroscopic image registration. Comp Aided Surg 1999; 4(2): 65-76

${ }^{5}$ Hofstetter R, Slomczykowski M, Sati M, Nolte P. Fluoroscopy as an imaging means for computed assisted surgical navigation. Comp Aided Surg 1999; 4(2): 65-76

${ }^{6}$ Jacob AL et al. Posterior pelvic ring fractures: closed reduction and percutaneous CT-guided sacroiliac screw fixation. Cardiovasc Intervent Radiol 1997; 20(4): 285-94

${ }^{7}$ Parker PJ et al. Percutaneous fluoroscopic screw fixation of acetabular fractures. Injury 1997; 28(9-10): 597-600

${ }^{8}$ Routt ML Jr et al. Early results of percutaneous ilisacral screws placed with the patient in the supine position. J Orthop Trauma 1995; 9(3): 207-14

${ }^{9}$ Routt ML Jr, Simonian PT. Closed reduction and percutaneous skeletal fixation of sacral fractures. Clin Orthop 1996; 329: $121-8$

${ }^{10}$ Shuler TE et al. Percutaneous ilisacral screw fixation; early treatment for unstable posterior pelvic ring disruptions. J Trauma 1995; 38(3): $453-8$
${ }^{11}$ Starr AJ et al. Percutaneous fixation of the columns of the acetabulum: a new technique. J Orthop Trauma 1998; 12(1): $51-8$

12 Stöckle U, König B, Hofstetter R et al. Bildwandler-gestützte Navigation: Eine experimentelle Studie zu Beckenverschraubungen. Unfallchirurg 2001; 104: 215-20

${ }^{13}$ Stöckle U, König B, Kandziora F et al. Clinical experiences with CT-based navigation in the thoracic spine. Comp Aid Surg 2001; 6: 120

${ }^{14}$ Suhm N, Jacob AL, Nolte LP et al. Surgical Navigation based on fluoroscopy: clinical application fot computer-assisted distal locking of intramedullary nails. Comp Aid Surg 2000; 5: $391-400$

\section{Priv. Doz. Dr. Ulrich Stöckle} Oberarzt

\section{Dr. med. Benjamin König}

Wiss. Mitarbeiter

Prof. Dr. med. Norbert Haas

Ärztl. Direktor

Unfall- und Wieder-

herstellungschirurgie

Humboldt-Universität zu Berlin

Charité. Campus Virchow-Klinikum Augustenburgerplatz 1

D-13353 Berlin 